\title{
AQUEOUS VEINS IN GLAUCOMATOUS EYES*
}

BY

\author{
T. L. Thomassen, E. S. Perkins, and J. H. Dobree \\ From the Institute of Ophthalmology, London. \\ Director of Research, Sir Stewart Duke-Elder
}

ONE of us has previously found (Thomassen, 1947b) that the aqueous veins in eyes suffering from simple glaucoma convey little or no clear liquid when the bulbar tension is in an increasing phase, whereas the clear outflow through them is augmented when the bulbar tension is in a decreasing phase. The main intention of this paper is to extend this observation by means of photographs: Gärtner (1944) and de Vries (1947) have already successfully photographed aqueous veins. Those accompanying this paper were taken by the Medical Illustration Department of the Institute of Ophthalmology.

\section{Method}

The bulbar tension was measured half hourly with a Schiötz X-tonometer. Before each measurement the eyes were anæsthetized with one drop 1 per cent. pantocain solution. By means of the repeated measurements it was possible to estimate whether the bulbar tension was increasing or decreasing. For further details concerning the estimation of the phases in the tension, the reader is referred to earlier explanations (Thomassen, 19.47a; Thomassen and Leydhecker, 1950). It is obvious that the repeated tonometry and the drops irritate the eye and therefore produce a generalized hyperæmia of the conjunctival vessels and aqueous veins. Observations and photographs have therefore been taken immediately before each new measurement, thus allowing time for the irritation from the previous measurements to subside.

In some cases the observation has been made difficult by another phenomenon. It has been found that the light from the slit-lamp can increase the amount of blood in the aqueous veins. In one case such a change was particularly striking. The patient, who had a simple glaucoma in both eyes, was observed on eight different days. In the left eye a large aqueous vein was seen with almost clear contents. When the light was switched on the speed of the current slowed down and after a short time the contents were rocking to and fro synchronously with the heart-beat. At the same time the vein was filling with blood. Finally, the contents of the vein, now only blood, were running quite freely in the direction opposite to that previously observed. After a short time in the dark the clear fluid was running as before, but

* Received for publication November 24, 1949. 
when the light was put on the same changes always took place. Sometimes the changes appeared after a few seconds, but sometimes a longer period of illumination, up to one minute or more, was necessary. The bulbar tension was not found to play any definite part in the phenomenon. Changes as dramatic as this have been observed only in this single case, but in some other cases a slightly increased amount of blood in the aqueous veins has been seen when the eyes have been exposed to bright light. These observations do not substantiate the findings in normal eyes of Ascher (1942b), that light increases the flow of clear liquid. It may be, however, that the phenomenon seen in our case is caused by the heat of the slit-lamp beam and not by the light itself.

\section{Results}

More than thirty patients with glaucoma have been examined, but most of the observations have been more or less equivocal. Only one case will therefore be described in detail. In this case the changes were very marked and we had the opportunity of making repeated observations so that the phenomena observed must be considered established.

This one patient, a woman 60 years old, had suffered from attacks of misty vision and haloes round lights for three years. The anterior chambers were rather shallow and the fields showed some peripheral contraction. She had never had an acute attack but nevertheless the disease could not be regarded as a simple glaucoma. She had used pilocarpine for one year, but no operation had been performed on her eyes. On the nasal side of the right eye was seen a large aqueous vein which originated near the limbus from several small tributaries. Fig. 1 shows the changes in tension in this eye from 10 a.m. to 4 p.m. on September 14, 1949 (no pilocarpine having been used for 36 hours). To begin with, the tension was in a decreasing phase and the contents of the aqueous vein appeared almost clear (Fig. 2). It should be noted that one drop of pantocain solution had been instilled into the eye and the bulbar tension measured once before this photograph was taken. Some minutes after the measurement at 10.30 a.m. it was observed that the vein was filled with blood, and from the curve it can be seen that the bulbar tension started rising. This appearance of the aqueous vein (Fig. 3) remained constant while the bulbar tension was in the increasing phase. It was remarkable that even the small tributaries were filled with blood and that the current had the same direction and about the same speed as before. The blood must therefore have displaced the aqueous intrasclerally, perhaps in Schlemm's canal. Unfortunately it was impossible to see this canal by gonioscopy on account of the shallowness of the anterior chamber. During the last 10 minutes before the measurement at 11.10 a.m. the vein became less engorged and the blood in the aqueous vein seemed to be a little diluted. After this mcasurement more clear liquid was again visible in the aqueous vein, particularly in the upper branches. and produced a marked stratification (Fig. 4). Unfortunately this photograph is not quite in focus, but a broad dilute stream in the upper part of the vein is visible. The pressure was now in a slightly decreasing phase and the aqueous vein appeared unchanged until, at about 2.00 p.m., it was again filled with blood and appeared the same as in Fig. 2 . The bulbar tension was now again in an increasing phase. As it was necessary to conclude the investigation within a limited time pilocarpine was instilled into the eye and as a consequence the tension again dropped. During this decreasing phase Fig. 5 was taken. This shows a marked stratification due to the presence of an increased amount of clear liquid in the upper part of the vein. 
The objection may still be advanced that the pantocain and -tonometry influence the results, in spite of the fact that the clear fluid in this case reappeared spontaneously after 4 drops of pantocaine and as many, measurements of the tension. This patient has been examined altogether 12 times in the course of 3 months. Each time no pilocarpine has been used during the previous 36 hours. The significant observation was made that the aqueous vein contained mainly clear liquid every morning. Between 10 and 11 a.m. the vein became filled with blood in the

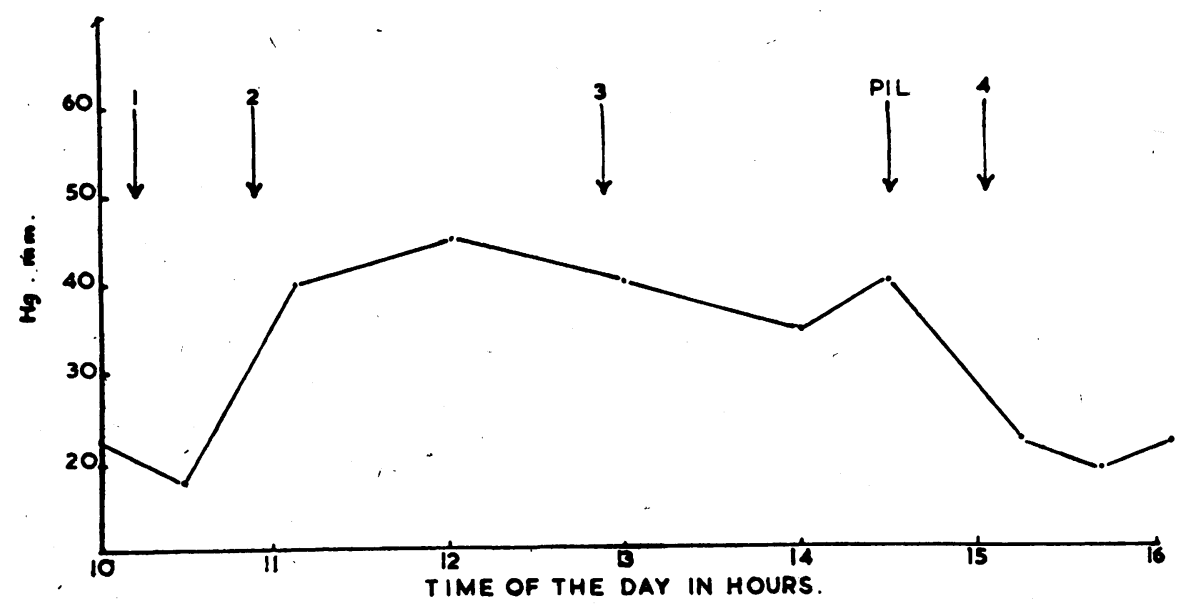

FIG. 1.-The oscillation of tension in a glaucomatous eye during one day. Pil. means that a 2 per cent. solution of pilocarpine is instilled in to the eye. Arrows 1 to 4 refer to the moments when Figs 2 to 5 were taken.

course of $10-15$ seconds. This alteration took place even if nothing were done to the eye except observation with the slit-lamp. Figs 6 and 7 were taken on such a day when no measurements had been made. Fig. 6 shows the same appearance as Fig. 2 and Fig. 7 is similar to Fig. 3. In Fig. 3 the aqueous vein in question is seen to be somewhat broader than in Fig. 6 . This may be caused by the drops and tonometry done before Fig. 3 was taken. In fact, when nothing was done to the eye, no hyperæmia was seen in the increasing phase except the filling of the aqueous veins with blood and some. dilatation of small veins joining it. Figs 8, 9, and 10 were taken by one of us (E. S. P.) on another day when no measurements of tension were performed. Fig. 8 was taken in the same phase as Fig. 2, Fig. 9 in the increasing phase (corresponding to Fig. 3), and Fig. 10 after the instillation of pilocarpine as in Fig. 5. The changes in this particular aqueous vein were indeed so regular that after a few days' observation we were able to tell by its appearance whether the bulbar tension was 
in an increasing or decreasing phase, and tonometry proved that we were never wrong. Only twice did the bulbar tension decrease again spontaneously after the pronounced increase. On other days the tension increased so much that pilocarpine had to be instilled.

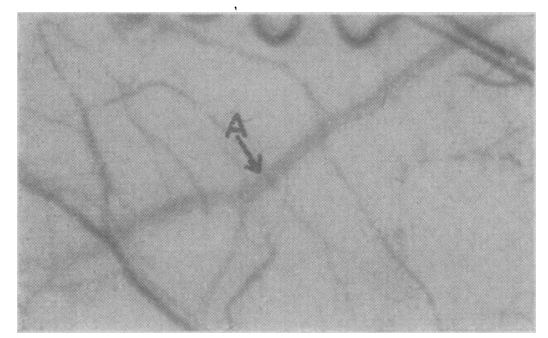

FI $r_{i} .2$

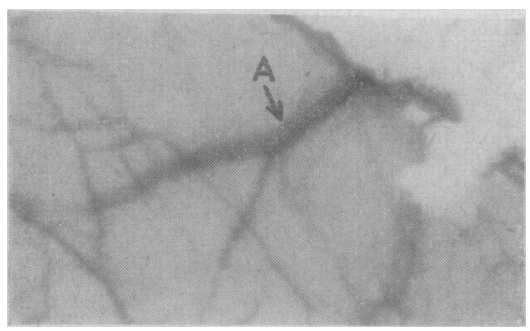

FiG. 4

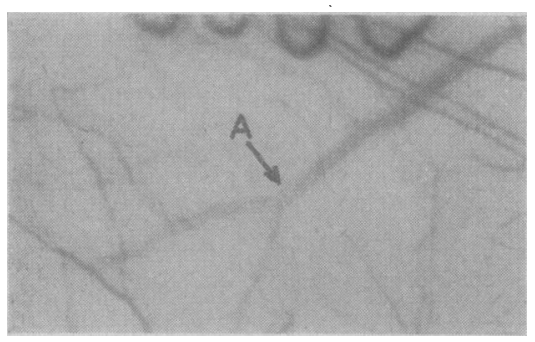

FIG. 6.

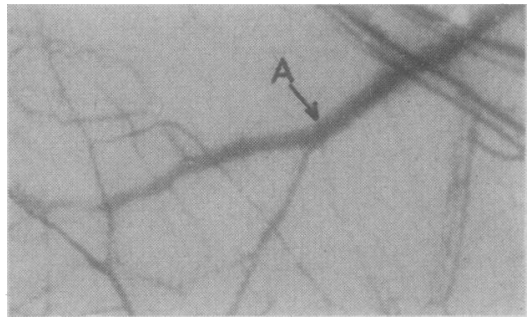

FIG. 3.

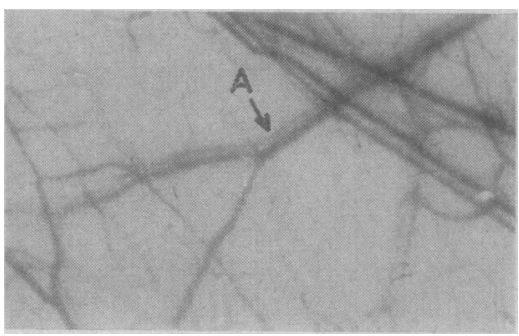

Fig. 5.

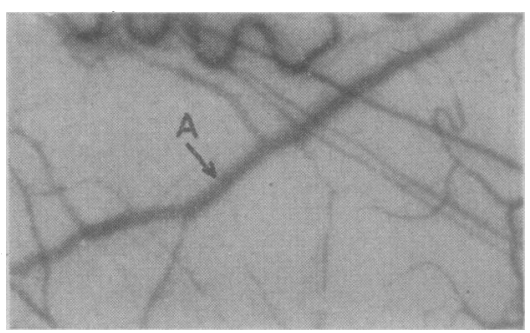

FIG. 7.

Figs 2-5 show the different appearances of the aqueous vein " A ". Figs 6 and 7 , taken on another day when no measurements of the tension were made, show the same aqueous vein "A ".

The observations on this case show that at least in some glaucomatous eyes there must be a close relationship between the appearance of the aqueous veins and the changes in the bulbar tension. There are three possible explanations of this relationship : 


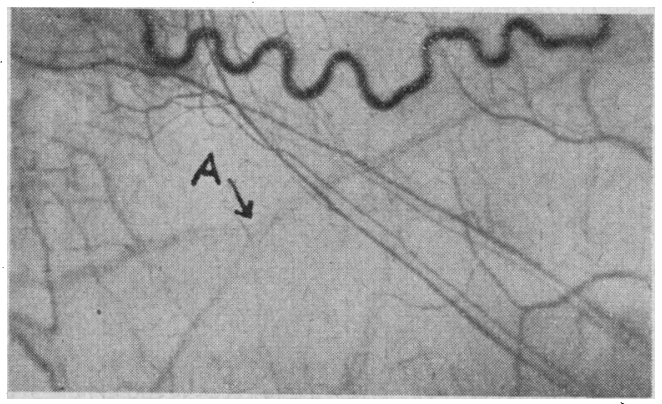

FIG. 8.

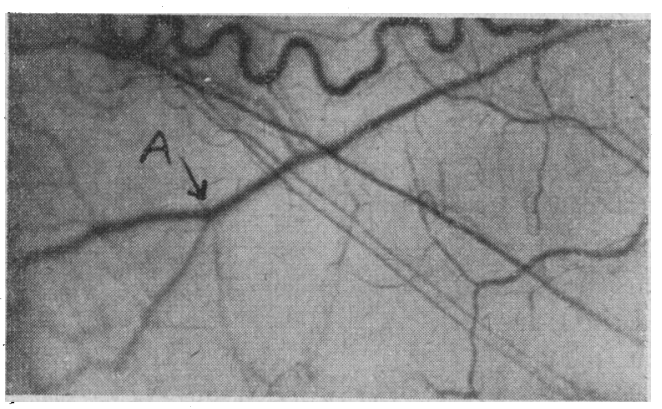

FIG. 9.

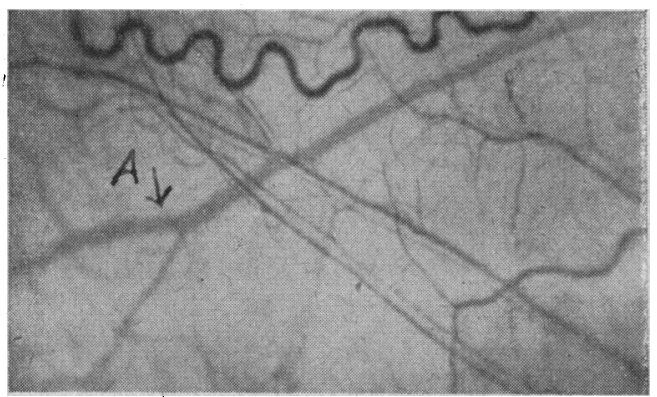

FIG. I0.

Figs 8, 9, and 10 showing the same aqueous vein " A" were taken some weeks later than 2-7, again without any measurements being made. Fig. 10 shows the vein " $A$ " after the instillation of pilocarpine.

(1) Thomassen (1947a) has emphasized that the venous pressure in the globe is high when the bulbar tension is increasing and low when it is decreasing. When the venous pressure is high it is easy to understand that the aqueous will 
be displaced by blood. The outflow of aqueous humour will therefore also be hampered, causing the bulbar tension to increase.

(2) Ascher and Spurgeon (1949) and Goldmann (1948) have emphasized that the pressure in the aqueous veins is decreased in glaucomatous eyes. This can of course explain our findings if it is supposed to occur only during the increasing phase.

(3) The third possible explanation is that there is no causative relation between the phenomena. That is to say that the increase of blood in the aqueous veins is a vascular phenomenon similar to the hyperæmia caused by irritation.

The pressure in some of the episcleral veins was measured by means of the water manometer described by Thomassen (1947a). In the first decreasing phase it was found to be about $5 \mathrm{~mm}$. Hg in the aqueous vein as well as in most of the other veins. When the bulbar tension started increasing the pressure in the aqueous vein and in the small veins connected with the aqueous vein also increased and rose by degrees to $14 \mathrm{~mm}$. $\mathrm{Hg}$. The rise in the venous pressure was not found to precede the rise in bulbar tension. Most of the other veins did not show any change in pressure. In some deep veins, however, marked changes were found, but again they did not precede the changes in the ocular tension. In any case the rapid change in the bulbar tension makes it very difficult to be sure that changes in the venous pressure precede the changes in ocular tension.

In conclusion, some observations on other glaucomatous eyes should be briefly mentioned. In some cases when the patient was first seen while the bulbar tension was in an increasing phase no aqueous vein could be found, although subsequent examination in the decreasing phase showed them to be present. In one case a marked narrowing of the blood column was seen during the increasing phase where the smaller veins entered the side of a larger vein. When the tension decreased again this could no longer be observed.

Gartner (1944) has shown that pilocarpine increases the outflow of clear liquid through the aqueous veins. Ascher (1942) has described the same phenomenon, but he finds it does not occur in every case. We can contirm their findings in so far as pilocarpine can increase the outflow, but only when it produces a decreasing phase in the bulbar tension. If pilocarpine does not produce this decrease in tension only a hyperæmia in the episcleral veins is seen and there is no alteration in the aqueous veins. 


\section{Summary}

The authors confirm by means of photographs the phenomenon previously described, that at least in some glaucomatous eyes the aqueous veins tend to be filled with blood when the bulbar tension is in an increasing phase and convey more clear liquid when the bulbar tension is decreasing.

\section{REFERENCES}

ASCHER, K. W. (1942a). Amer.J. Ophthal., 25, 1174.

(1942b). Ibid., 25, 1301 .

and SPURGEON, IV. M. (1949). Ibid., 32, 239.

Gartner, S. (1944). Arch. Ophthal.. Chicago, 32, 464.

Goldmann, H. (1948). Ophtha/mologica, 116, 195.

THOMASSEN, T. 1.. (1947a). Acta ophthal., Kbh.. 25, 221.

(1947b). Ibid., 25, 369.

and LEYDHECKER, W. (1950). British Journal of Ophthalmology, 34, 169.

DE VRIEs, S. (1947). " De Zichtbare Afvoervan het Kamerwater." Amsterdam. 